ISSN 0103-5150

Fisioter. Mov., Curitiba, v. 26, n. 2, p. 361-368, abr./jun. 2013

Licenciado sob uma Licença Creative Commons

\title{
Caracterização de lesões desportivas no basquetebol
}

\author{
Characterization of sport injuries in the basketball
}

\section{Antônio Francisco de Almeida Neto ${ }^{[a]}$, Juliana Petrongari Tonin ${ }^{[b]}$, Marcelo Tavella Navega ${ }^{[c]}$}

[a] Mestrando em Desenvolvimento Humano e Tecnologias, Instituto de Biociências, Departamento de Educação Física, Universidade Estadual Paulista (Unesp), Rio Claro, SP - Brasil, e-mail: tonholabiomecrc@yahoo.com.br

[b] Discente do curso de Fisioterapia, Faculdade de Filosofia e Ciências, Departamento de Educação Especial, Universidade Estadual Paulista (Unesp), Marília, SP - Brasil, e-mail: ju.tonin@hotmail.com

[c] Docente do curso de Fisioterapia, Faculdade de Filosofia e Ciências, Departamento de Educação Especial da Universidade Estadual Paulista (Unesp), Marília, SP - Brasil, e-mail: marcelonavega@yahoo.com.br

\section{Resumo}

Introdução: $O$ basquete apresenta altos índices de lesão. Na literatura ainda não se definiu existem ou não diferenças entre os sexos. Objetivo: Caracterizar e analisar a incidência de lesões desportivas em atletas de basquetebol, com comparação entre os sexos. Materiais e métodos: Quinze atletas de cada sexo, da categoria sub-23, foram entrevistados com o inquérito de morbidade referida. Resultados: A média de idade das equipes feminina e masculina foi de $18 \pm 0,65$ e 18,20 \pm 1,57 anos, respectivamente. A equipe masculina apresentou mais lesões que a feminina $(2,6 \pm 1,45$ contra $1,2 \pm 1,18$ respectivamente, $p<0,05)$. A lesão articular foi o tipo de lesão mais comum na equipe masculina, representando 58,97\%, e o segundo mais comum na feminina $(33,33 \%)$. Os membros inferiores foram a região mais acometida por lesões $(80,95 \%$ na feminina e $69,23 \%$ na masculina). 0 mecanismo de lesão mais comum na equipe masculina foi a aterrissagem $(43,59 \%)$ e na feminina o salto vertical $(28,57 \%)$. Em ambas as equipes, a maior parte das lesões ocorreu durante os treinos (61,9\% na feminina e 71,8\% na masculina). Foi necessário o afastamento em 47,62\% (feminina) e 56,41\% (masculina). A maioria dos atletas não realizou tratamento $(61,9 \%$ e 51,28\% feminino e masculino, respectivamente). 0 retorno às atividades, na maioria das vezes, foi sintomático, tanto na equipe feminina quanto na masculina $(85,71 \%$ e $84,62 \%$ respectivamente). Conclusão: A incidência de lesões no 
basquetebol é maior nos membros inferiores. A equipe masculina mostra-se mais propensa a sofrer lesões, e os principais mecanismos de lesão foram diferentes entre as equipes masculina e feminina.

Palavras-chave: Lesões esportivas. Basquetebol. Fisioterapia (especialidade).

\section{Abstract}

Introduction: Basketball is characterized with high injury rate. In the literature it's not defined whether or not there are differences between the sexes. Objective: Characterize and analyze the incidence of athletic injuries in basketball players, comparing the sexes. Materials and methods: Fifteen under-23 category athletes of each sex were interviewed with the reported morbidity inquire. Results: The age of the female and male teams, in average, was $18 \pm 0.65$ and $18.20 \pm 1.57$ years respectively. The male team had more injuries than the female one (respectively $2.6 \pm 1.45$ against $1.2 \pm 1.18, p<0.05$ ). The joint damage was the most common injury in the male team, representing 58.97\%, and the second most common in the female team (33.33\%). The lower limbs were the most common injured sites (80.95\% in the female and $69.23 \%$ in the male). The most frequent injury mechanism in the male team was the landing (43.59\%), and in the female was the vertical jump (28.57\%). In both teams the most injuries occurred during the training (61.9\% in the female and $71.8 \%$ in the male). The absence was necessary in $47.62 \%$ (female) and $56.41 \%$ (male). Great part of the athletes didn't received any treatment (61.9\% and $51.28 \%$ in the female and male team respectively). The return to the activities was mostly symptomatic, in both female and male team ( $85.71 \%$ and $84.62 \%$ respectively). Conclusion: The incidence of injuries in basketball is higher in the lower limbs. The male team is more likely to suffer injuries, and the main injury mechanisms were different between male and female team.

Keywords: Athletic injuries. Basketball. Physical therapy (specialty).

\section{Introdução}

0 basquete é uma modalidade esportiva que envolve esforços intensos e breves, exigindo grande movimentação e coordenação $(1,2)$. Esse esporte apresenta alta incidência de lesões, causadas por frequentes saltos, aterrissagens, mudanças de direção, pivoteios e contato físico característicos de sua prática (3-6).

Aliado a esses fatores, a solicitação por aprimoramento físico, técnico e tático, resultante do treinamento desportivo de alto desempenho, se associa com desequilíbrios físicos e biomecânicos variados, como retrações musculares, desalinhamentos posturais, além de lesões desportivas (LD) (7-11).

Quando é levado em consideração o sexo, existem controvérsias quanto à maior incidência de lesões. Em estudo de Waterman, Belmont, Cameron, Svoboda, Alitz e Owens (12), o sexo masculino é considerado fator de risco para LD, enquanto que nos estudos de Kofotolis e Kellis (4), Oliveira, Bang, Quitério, Padovani e Oliveira (13), Weinhandl, Joshi e O'Connor (14), Borowski, Yard, Fields e Comstock (15), de Loës, Dahlstedt e Thomée (16) e Messina,
Farney e DeLee (17) a maior incidência de lesão ocorreu em atletas do sexo feminino. Em contrapartida, Moreira (18) e Nelson, Yard, Fields e Comstock (19) não encontraram diferenças entre os sexos.

0 objetivo do presente estudo foi caracterizar e analisar a incidência de LD em atletas de basquetebol, com comparação entre os sexos.

\section{Métodos}

O estudo foi aprovado pelo Comitê de Ética em Pesquisa da Faculdade de Filosofia e Ciências da Universidade Estadual Paulista (Unesp), Câmpus Marília, mediante protocolo n. 1354/2010. Participaram do estudo sujeitos que assinaram o Termo de Consentimento Livre e Esclarecido. Em casos de sujeitos menores de idade, os responsáveis assinaram o termo de consentimento.

Foram avaliados no total 30 atletas praticantes de basquetebol há pelo menos dois anos, categoria sub-21; 15 eram da equipe masculina do Yara Clube e 15, da equipe feminina do Colégio Cristo Rei; ambas as equipes eram da cidade de Marília (SP). A coleta de dados foi 
realizada individualmente em sala anexa à quadra de treinamento, em horários pré-agendados com os atletas.

Para caracterização dos sujeitos do estudo, foram coletadas informações relativas a sexo, massa corporal, estatura, índice de massa corporal (IMC), tempo de treinamento (horas semanais e anos de treino), dominância de membros e posição em que joga.

Para a obtenção dos dados relativos às lesões foi utilizado o Inquérito de Morbidade Referida (IMR) (9). Foram feitas adaptações ao questionário para que este se tornasse mais específico ao basquetebol (IMRb) (Apêndice A). Este inquérito já foi adaptado para outros esportes, como o taekwondo (13), a ginástica artística (20), o tênis de mesa (21) e a natação (22). A parte inicial deste instrumento destina-se à obtenção de dados pessoais e características físicas do atleta (sexo, idade, estatura, massa corporal, tempo de treinamento e presença de lesão). Foram, na sequência, coletados os dados referentes ao tipo de lesão, local anatômico, período de treinamento, mecanismo de lesão ou aumento do sintoma, tratamento, tempo de afastamento e retorno às atividades normais. Questionários são considerados importantes ferramentas clínicas e de pesquisa, uma vez que são eficientes, tem baixo custo e boa confiabilidade (23). A pesquisa foi realizada levando-se em conta a última temporada, ou seja, 18 meses anteriores à data da coleta.

Os itens referentes ao tipo de lesão/sintomas do IMRb foram agrupados nas seguintes categorias: lesões articulares (entorse, sinovite, bursite, luxação e subluxação); lesões musculares (contusão, distensão muscular, contratura muscular e mialgia); lesões tendíneas (tendinopatia); lesões ósseas (periostite e fratura) e outras lesões (dor aguda inespecífica, dor crônica inespecífica e outras). De forma semelhante, os itens do domínio local anatômico foram agrupados em: tronco (tórax, abdome, região cervical, região torácica e região lombar); membros superiores (ombro, braço, antebraço, cotovelo, punho e mão); membros inferiores (quadril, virilha, coxa anterior, coxa superior, coxa medial, coxa lateral, joelho, perna, panturrilha, tornozelo e pé) e outras regiões (cabeça e outras).

Para a análise dos dados, foram utilizadas técnicas de estatística descritiva e analítica. Foi realizado o teste de Shapiro-Wilk para verificar a normalidade dos dados. Para as comparações entre os grupos, foi realizado o teste de Mann-Whitney. Para a comparação de variáveis dicotômicas nominais foi usado o teste do Qui-quadrado $\left(\mathrm{x}^{2}\right)$. Para as interpretações dos resultados estatísticos, foi adotado o nível de significância de $5 \%$ (p $\leq 0,05)$.

\section{Resultados}

As características dos atletas estão apresentadas na Tabela 1. Não foram encontradas diferenças entre os grupos no que diz respeito à idade. Foram observadas diferenças quanto a massa corporal, estatura e IMC.

A média de tempo de treinamento foi de 6,67 \pm 3,48 anos para a equipe feminina, e 5,40 $\pm 2,80$ anos para a equipe masculina. 0 tempo de treinamento semanal foi de 8,13 $\pm 0,3$ horas para a equipe feminina e $10,07 \pm 3,58$ para a equipe masculina. Os grupos não apresentaram diferenças entre si em nenhuma dessas variáveis.

0 número de atletas avaliados da equipe feminina/masculina por posição foi: ala, 5/7; pivô, 5/6; armador, 2/1; ala armador, 2/0; ala pivô 1/1.

Dos 15 atletas do sexo masculino, apenas um não relatou ter sofrido lesão, enquanto que, das 15 atletas femininas, quatro não apresentaram lesões. No total, foram relatadas $60(100 \%)$ lesões, 39 (65\%) na equipe masculina e 21 (35\%) na equipe feminina. A equipe masculina apresentou mais lesões que a feminina $(\mathrm{p}<0,05)$. Em média, a equipe masculina apresentou $2,6 \pm 1,45$ lesões e a feminina $1,2 \pm 1,18$ lesões.

0 tipo de lesão mais comum na equipe masculina foi a lesão articular, representando $58,97 \%$ do total de lesões deste grupo. Destaca-se o entorse de tornozelo, que foi o tipo de lesão articular mais relatado na equipe masculina (das 23 lesões articulares, 22 foram entorses de tornozelo). Já na equipe feminina, a lesão articular foi o segundo tipo mais frequente $(33,33 \%)$. Das sete lesões articulares, quatro foram entorses de tornozelo. Os demais tipos de LD apresentados estão expostos na Tabela 2. Aplicando-se o teste do Qui-quadrado observa-se que o sexo teve influência no tipo da lesão $\left(x^{2}=15,17\right.$ e p $\left.=0,0043\right)$. Os subtipos de lesão específicos estão representados na Figura 1.

Levando-se em conta o local em que ocorreram as lesões, os membros inferiores foram os mais acometidos em ambas as equipes, representando 80,95\% das lesões da equipe feminina e $69,23 \%$ da equipe masculina. Os membros superiores representaram $19,05 \%$ das lesões na equipe feminina e $25,64 \%$ da masculina, enquanto que a masculina apresentou $5,13 \%$ das suas lesões no tronco. Por meio do teste do Qui-quadrado, nota-se que o sexo teve influência nos locais em que ocorreram lesões $\left(x^{2}=23,5\right.$ e $p=$ 0,015). Os locais anatômicos específicos são mostrados na Figura 2. 
Tabela 1 - Características dos atletas

\begin{tabular}{lcccc}
\hline Sexo & Idade (anos) & Massa corporal $(\mathbf{k g})$ & Estatura $(\mathrm{m})$ & IMC $\left(\mathbf{k g} / \mathbf{m}^{2}\right)$ \\
\hline Feminino $(\mathbf{n}=\mathbf{1 5})$ & $18 \pm 0,65$ & $60,7 \pm 6,90^{\star}$ & $1,72 \pm 0,06^{\star}$ & $20,32 \pm 1,46^{* *}$ \\
Masculino $(\mathbf{n}=\mathbf{1 5})$ & $18,20 \pm 1,57$ & $84,26 \pm 14,28$ & $1,88 \pm 0,06$ & $23,87 \pm 3,11$ \\
\hline
\end{tabular}

Legenda: $\mathrm{n}$ = número de sujeitos; $\mathrm{kg}$ = quilograma; $\mathrm{m}=$ metros; $\mathrm{IMC}=$ Índice de Massa Corporal; $\mathrm{kg} / \mathrm{m}^{2}$ = quilograma por metro quadrado; * = diferença significativa entre 0 grupo feminino e masculino, $p<0,0001 ;{ }^{* *}=$ diferença significativa entre 0 grupo feminino e masculino, $p<0,01$.

Fonte: Dados da pesquisa.

Tabela 2 - Tipos de lesão mais comuns

\begin{tabular}{lcccccccccc}
\hline Sexo & Lesões articulares & \multicolumn{2}{c}{ Lesões musculares } & \multicolumn{2}{c}{ Lesões tendíneas } & Lesões ósseas & \multicolumn{2}{c}{ Outras lesões } \\
\hline \multirow{3}{*}{ Feminino } & $\mathrm{n}$ & $\%$ & $\mathrm{n}$ & $\%$ & $\mathrm{n}$ & $\%$ & $\mathrm{n}$ & $\%$ & $\mathrm{n}$ & $\%$ \\
Masculino & 7 & 33,33 & 6 & 28,57 & - & - & - & - & 8 & 38,10 \\
& 23 & 58,97 & 7 & 17,95 & 4 & 10,26 & 3 & 7,69 & 2 & 5,13 \\
\hline
\end{tabular}

Legenda: $\mathrm{n}$ = número absoluto de lesões; \% = porcentagem referente ao total de lesões de cada sexo.

Fonte: Dados da pesquisa.

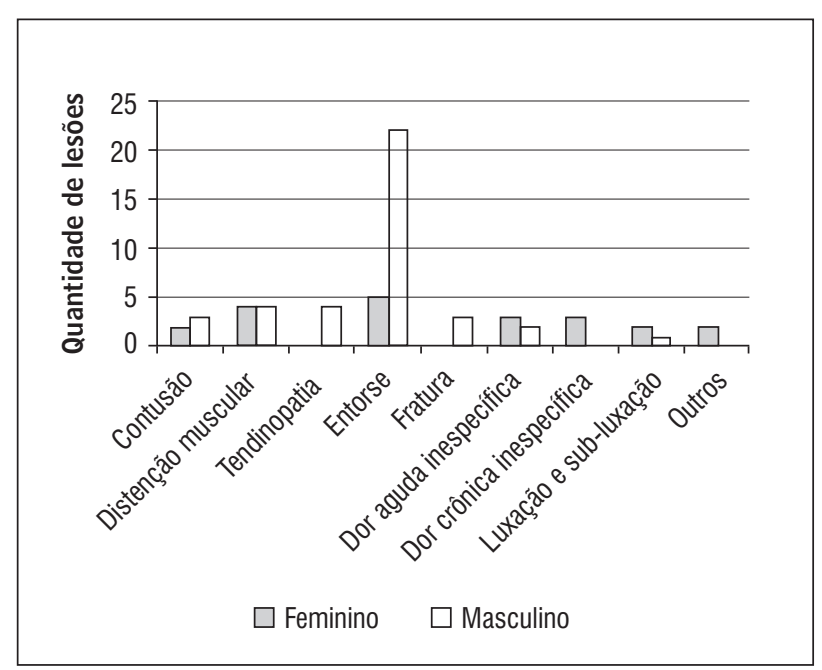

Figura 1 - Subtipos de lesão

Fonte: Dados da pesquisa.

O mecanismo de lesão mais comum na equipe masculina foi a aterrissagem $(43,59 \%)$, e na feminina foi o salto vertical $(28,57 \%)$. A Tabela 3 mostra todos os mecanismos de lesão considerados e a sua incidência.

A maioria das lesões, tanto na equipe feminina $(61,9 \%)$ quanto na masculina $(71,8 \%)$ ocorreram durante os treinos; não houve diferença entre os

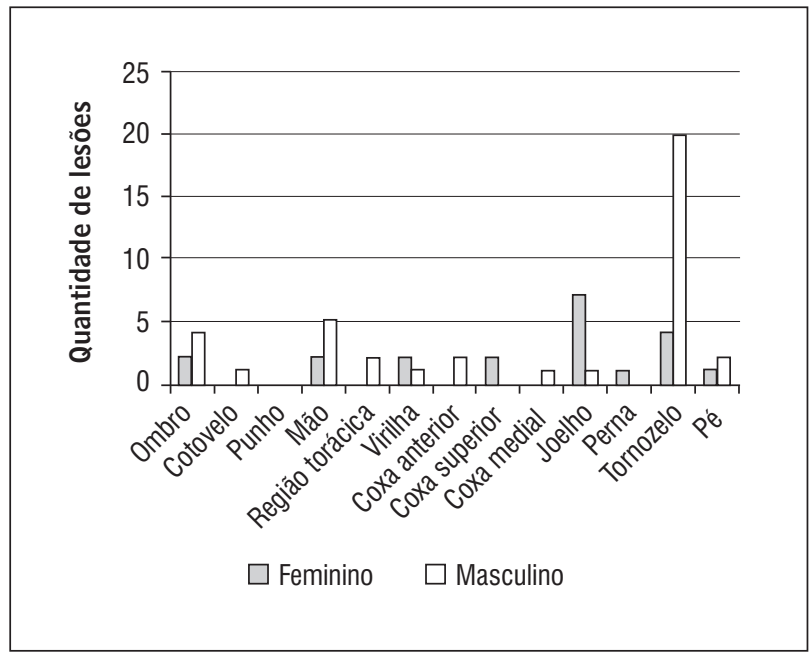

Figura 2 - Locais anatômicos em que ocorreram lesões Fonte: Dados da pesquisa.

grupos $\left(\mathrm{x}^{2}=0,8424\right.$ e $\left.\mathrm{p}=0,3587\right)$. Foi necessário o afastamento dos atletas em $47,62 \%$ das lesões da equipe feminina e $56,41 \%$ da masculina. Na maior parte das vezes, os atletas não realizaram nenhum tipo de tratamento $(61,9 \%$ e $51,28 \%$, equipe feminina e masculina, respectivamente) e o retorno às atividades foi, em grande parte, sintomático $(85,71 \%$ na equipe feminina e $84,62 \%$ na equipe masculina). 
Tabela 3 - Mecanismo de lesão

\begin{tabular}{|c|c|c|c|}
\hline & & Feminino & Masculino \\
\hline Aterrissagem & $\begin{array}{l}\mathrm{n} \\
\%\end{array}$ & $\begin{array}{c}1 \\
4,76\end{array}$ & $\begin{array}{c}17 \\
43,59\end{array}$ \\
\hline Salto vertical & $\begin{array}{l}n \\
\%\end{array}$ & $\begin{array}{c}6 \\
28,57\end{array}$ & - \\
\hline Choque & $\begin{array}{l}n \\
\%\end{array}$ & $\begin{array}{c}2 \\
9,52\end{array}$ & $\begin{array}{c}5 \\
12,82\end{array}$ \\
\hline Explosão & $\begin{array}{l}n \\
\%\end{array}$ & $\begin{array}{c}3 \\
14,29\end{array}$ & $\begin{array}{c}4 \\
10,26\end{array}$ \\
\hline Corrida de velocidade & $\begin{array}{l}n \\
\%\end{array}$ & $\begin{array}{c}3 \\
14,29\end{array}$ & $\begin{array}{c}3 \\
7,69\end{array}$ \\
\hline Arremesso & $\begin{array}{l}n \\
\%\end{array}$ & $\begin{array}{c}1 \\
4,76\end{array}$ & $\begin{array}{c}2 \\
5,13\end{array}$ \\
\hline Queda & $\begin{array}{l}n \\
\%\end{array}$ & $\begin{array}{c}1 \\
4,76\end{array}$ & $\begin{array}{c}2 \\
5,13\end{array}$ \\
\hline Musculação & $\begin{array}{l}n \\
\%\end{array}$ & - & $\begin{array}{c}2 \\
5,13\end{array}$ \\
\hline Lançamento (passe) & $\begin{array}{l}n \\
\%\end{array}$ & $\begin{array}{c}1 \\
4,76\end{array}$ & - \\
\hline Outros mecanismos & $\begin{array}{l}\mathrm{n} \\
\%\end{array}$ & $\begin{array}{c}3 \\
14,29\end{array}$ & $\begin{array}{c}4 \\
10,26\end{array}$ \\
\hline
\end{tabular}

Legenda: $\mathrm{n}=$ número absoluto de lesões; $\%$ = porcentagem referente ao total de lesões de cada sexo.

Fonte: Dados da pesquisa.

\section{Discussão}

No presente estudo foi investigada a incidência e a caracterização de lesões em atletas de basquetebol de ambos os sexos.

Foi observado que ocorreram mais lesões na equipe masculina do que na feminina, provavelmente por causa das características físicas que tornam maior a intensidade do jogo na categoria masculina (12). A maioria das lesões de ambos os grupos ocorreu nos membros inferiores, o que é explicado pelas características de descarga de peso do jogo, que inclui corrida, mudança rápida de direção, saltos e aterrissagens $(3-7,18,24-27)$. Levando-se em conta as equipes masculina e feminina, a lesão mais comum foi o entorse, e o local anatômico foi o tornozelo. Existem diversos estudos na literatura que apontam esta lesão como sendo a mais comum entre praticantes amadores e profissionais de diversos esportes, inclusive o basquetebol (28-36).

Quando separados os sexos, a equipe masculina apresentou a maioria das lesões no tornozelo e a equipe feminina apresenta maior ocorrência de lesões no joelho, o que corrobora com dados da literatura (14, 16, 17, 37-39). Em seu estudo, Fagenbaum e Darling (40) observaram que o padrão de ativação muscular do vasto lateral, vasto medial e isquiotibiais foi semelhante para jogadores de basquete de ambos os sexos; sendo assim, a maior propensão do sexo feminino a lesões no joelho é multifatorial, dentre os fatores estão estatura, massa corporal, índice de massa corporal, flexibilidade e força muscular, maior frouxidão ligamentar no joelho e generalizada $(37,41-43)$.

0 mecanismo de lesão mais recorrente na equipe feminina foi o salto vertical (28,57\%), e na masculina foi a aterrissagem $(43,59 \%)$. Esses dois mecanismos foram apontados por Borowski et al. (15) como frequentes causadores de lesões ligamentares. É comum ocorrerem lesões no basquete, dentre elas o entorse de tornozelo, resultantes da aterrissagem sobre o pé de outro jogador $(44,45)$. Quanto à maior incidência de lesões na equipe feminina durante o salto vertical, sugere-se que como causa o alto número de lesões musculares, uma vez que o salto se inicia com contração excêntrica da musculatura.

O maior número de lesões ocorreu durante o treino. 0 fato de os atletas passarem mais tempo treinando do que competindo pode explicar esse achado (7). Entretanto, existem estudos que divergem desse resultado,alegando que o aumento da intensidade de jogo nas competições é responsável por ocorrerem mais lesões nas competições $(6,19,26,28)$. Essa divergência pode ser explicada pelo fato de alguns estudos realizarem o cálculo do índice de lesão em treinos e competições, dessa forma, o índice de lesão em competições pode ficar maior que o dos treinos, uma vez que os atletas passam menos dias competindo do que treinando (6).

0 retorno dos atletas às atividades foi na, maior parte das vezes, sintomático; os atletas não realizaram nenhum tratamento. Em razão da necessidade de retorno rápido à prática esportiva, programas de prevenção são de grande importância para evitar o afastamento dos atletas por ocorrência de lesões.

Conclui-se que a incidência de lesões no basquetebol é maior nos membros inferiores. A equipe masculina mostra-se mais propensa a sofrer lesões, e os principais mecanismos de lesão foram diferentes entre as equipes masculina e feminina, o que mostra que o trabalho de prevenção tem que ser realizado especificamente para cada categoria, visando maior efetividade na diminuição da incidência de lesões. 


\section{Referências}

1. Moreira P, Gentil D, Oliveira C. Prevalence of injuries of Brazilian basketball national team during 2002 season. Rev. Bras. Med. Esporte. 2003;9(5):263-66.

2. Sacco ICN, Takahasi HY, Vasconcelos AA, Suda EY, Bacarin TA, Pereira CS, et al. Influência de implementos para o tornozelo nas respostas biomecânicas do salto e aterrissagem no basquete. Rev. Bras. Med. Esporte. 2004;10(6):447-52. doi:10.1590/ S1517-86922004000600001.

3. Acquesta FM, Peneireiro GM, Bianco R, Amadio AC, Serrão JC. Características dinâmicas de movimentos seleccionados do basquetebol. Rev Port Cien Desp. 2007;7(2):174-82.

4. Kofotolis N, Kellis E. Ankle sprain injuries: a 2-year prospective cohort study in female Greek professional basketball players. J Athl Train. 2007;42(3):388-94. PMid:18059995.

5. Vamvakoudis E, Vrabas IS, Galazoulas C, Stefanidis P, Metaxas TI, Mandroukas K. Effects of basketball training on maximal oxygen uptake, muscle strength, and joint mobility in young basketball players. J. Strength Cond. Res. 2007;21(3):930-36. doi:10.1519/R-18435.1.

6. Silva AS, Abdalla RJ, Fisberg M. Incidência de lesões musculoesqueléticas em atletas de elite do basquetebol feminino. Acta Ortop. Bras. 2007;15(1):43-6. doi:10.1590/S1413-78522007000100009.

7. Gantus MC, Assumpção JD. Epidemiologia das lesões do sistema locomotor em atletas de basquetebol. Acta Fisiátrica. 2002;9(2):77-84.

8. Pieter W, Heijmans J. Training \& Competition in Taekwondo. J. Asian Martial Arts. 2003;13(1):8-23

9. Pastre CM, Carvalho G Filho, Monteiro HL, Netto J Jr, Padovani CR. Lesões desportivas no atletismo: comparação entre informações obtidas em prontuários e inquéritos de morbidade referida. Rev. Bras. Med. Esporte. 2004;10(1):1-8. doi:10.1590/ S1517-86922004000100001.

10. Pastre CM, Carvalho G Filho, Monteiro HL, Netto J Jr, Padovani CR. Lesões desportivas na elite do atletismo brasileiro: estudo a partir de morbidade referida. Rev. Bras. Med. Esporte. 2005;11(1):43-47. doi:10.1590/ S1517-86922005000100005.
11. Pastre CM, Carvalho G Filho, Monteiro HL, Netto J Jr, Padovani CR, García AB. Exploração de fatores de risco para lesões no atletismo de alta performance. Rev. Bras. Med. Esporte. 2007; 13(3):200-04. doi:10.1590/ S1517-86922007000300014.

12. Waterman BR, Belmont PJ Jr, Cameron KL, Svoboda SJ, Alitz CJ, Owens BD. Risk factor for syndesmotic and medial ankle sprain: role of sex, sport, and level of competition. Am. J. Sports Med. 2011; ahead of print

13. Oliveira MR, Bang FSJ, Quitério RJ, Padovani CR, Oliveira AS Jr. Incidência e caracterização de lesões desportivas no Taekwondo segundo o sexo. Revista Inspirar. 2010;2(4):33-41.

14. Weinhandl JT, Joshi M, O'Connor KM. Gender comparisons between unilateral and bilateral landings. J. Appl. Biomech. 2010;26(4):444-53.

15. Borowski LA, Yard EE, Fields SK, Comstock RD. The epidemiology of US high school basketball injuries, 2005-2007. Am. J. Sports. Med. 2008;36(12):2328-35. doi:10.1177/0363546508322893.

16. de Löes M, Dahlstedt LJ, Thomée R. A 7-year study on risks and costs of knee injuries in male and female youth participants in 12 sports. Scand. J. Med. Sci. Sports. 2000;10(2):90-97. doi:10.1034/ j.1600-0838.2000.010002090.x.

17. Messina DF, Farney WC, DeLee JC. The incidence of injury in Texas high school basketball: a prospective study among male and female athletes. Am. J. Sports. Med. 1999; 27(3):294-99. PMid:10352762.

18. Moreira P. Prevalência de lesões das equipes de base e adultas que representaram a Seleção Brasileira de Basquete em 2003. R. Bras. Ciênc. Mov. 2006; 14(2):71-8.

19. Nelson AJ, Collins CL, Yard EE, Fields SK, Comstock RD. Ankle injuries among United States high school sports athletes, 2005-2006. J. Athl. Train. 2007;42(3):381-87.

20. Hoshi RA, Pastre CM, Vanderlei LCM, Netto J Jr, Bastos FN. Lesões desportivas na ginástica artística: estudo a partir de morbidade referida. Rev. Bras. Med. Esporte. 2008;14(5):440-45. doi:10.1590/ S1517-86922008000500008.

21. Silva JAMG, Almeida AF Neto, Oliveira MR, Faganello FR. Caracterização das lesões desportivas em atletas de tênis de mesa. Ter Man. 2010;8(40):430-34. 
22. Aguiar PRC, Bastos FN, Nascimento F Netto Júnior, Vanderlei LCM, Pastre CM. Lesões desportivas na natação. Rev Bras Med Esporte. 2010;16(4):273-77. doi:10.1590/S1517-86922010000400008.

23. Noronha M, Refshauge KM, Kilbreath SL, Figueiredo VG. Cross-cultural adaptation of the Brazilian-Portuguese version of the Cumberland Ankle Instability Tool (CAIT). Disabil Rehabil. 2008;30(26):1959-65. doi:10.1080/09638280701809872.

24. McKay GD, Goldie PA, Payne WR, Oakes BW, Watson LF. A prospective study of injuries in basketball: a total profile and comparison by gender and standard of competition. J Sci Med Sport. 2001;4(2):196-211. doi:10.1016/S1440-2440(01)80030-X.

25. Starkey C. Injuries and illness in the National Basketball Association: a 10-year perspective. J. Athl. Train. 2000;35(2):161-67. PMid:16558626.

26. Kujala UM, Taimela S, Antti-Poika I, Orava S, Tuominem R, Myllynen P. Acute injuries in soccer, ice hockey, volleyball, basketball, judo, and karate: analysis of national registry data. Br J Sports Med. 1995;311(7018):1465-68.

27. Burnham BR, Copley GB, Shim MJ, Kemp PA. Mechanism of basketball injuries reported to the HQ Air Force Safety Center: A 10-year descriptive study, 1993-2002. Am J Prev Med. 2010;38(S1):S134-40. doi:10.1016/j.amepre.2009.10.009.

28. Brown C, Padua D, Marshall SW, Guskiewicz K. Individuals with mechanical ankle instability exhibit different motion patterns than those with functional ankle instability and ankle sprain copers. Clin. Biomech. 2008;23(6):822-32. doi:10.1016/j. clinbiomech.2008.02.013.

29. Le Gall F, Carling C, Reilly T. Injuries in young elite female soccer players: an 8-season prospective study. Am J Sports Med. 2008;36(2):276-84. doi:10.1177/0363546507307866.

30. Mitchell A, Dyson R, Hale T, Abraham C. Biomechanics of ankle instability. Part 2: Postural sway-reaction time relationship. Med. Sci. Sports Exerc. 2008;40(8):152228. doi:10.1249/MSS.0b013e31817356d6.
31. Pedowitz DI, Reddy S, Parekh SG, Huffman GR, Sennett BJ. Prophylactic bracing decreases ankle injuries in collegiate female volleyball players. Am J Sports Med. 2008;36(2):324-27. doi:10.1177/ 0363546507308358.

32. Sekir U, Yildiz Y, Hazneci B, Ors F, Saka T, Aydin T. Reliability of a functional test battery evaluating functionality, proprioception, and strength in recreational athletes with functional ankle instability. Eur J Phys Rehabil Med. 2008;44(4):407-15. PMid:19002090.

33. Hubbard TJ, Cordova M. Mechanical instability after an acute lateral ankle sprain. Arch Phys Med Rehabil. 2009;90(7):1142-46. doi:10.1016/j. apmr.2009.01.020.

34. Hupperets MDW, Verhagen EALM, Van Mechelen W. Effects of sensorimotor training on morphological, neurophysiological and functional characteristics of the ankle: a critical review. Sports Med. 2009;39(7):591605. doi:10.2165/00007256-200939070-00005.

35. Yard EE, Collins CL, Comstock RD. A comparison of high school sports injury surveillance data reporting by certified athletic trainers and coaches. J Athl Train. 2009;44(6):645-52. doi:10.4085/ 1062-6050-44.6.645.

36. Walls RJ, Brennan SA, Hodnett P, O’byrne JM, Eustace SJ, Stephens MM. Overuse ankle injuries in professional Irish dancers. Foot Ankle Surg. 2010;16(1):45-49. doi:10.1016/j.fas.2009.05.003.

37. Myer GD, Ford KR, Paterno MV, Nick TG, Hewett TE. The effects of generalized joint laxity on risk of anterior cruciate ligament injuries in young female athletes. Am J Sports Med. 2008;36(6):1073-80. doi:10.1177/0363546507313572.

38. Myklebust G, Engebresten L, Braekken IH, Skjølberg A, Olsen O-E, Bahr R. Prevention of anterior cruciate ligament injuries in female team handball players: a prospective intervention study over three seasons. Clin J Sport Med. 2003;13(2):71-78. doi:10.1097/00042752-200303000-00002.

39. Hewett TE, Ford KR, Myer GD. Anterior cruciate ligament injuries in female athletes: part 2, a meta-analysis of neuromuscular interventions aimed at injury prevention. Am. J. Sports. Med. 2006;34(3):490-98. doi:10.1177/0363546505282619. 
40. Fangerbaum R, Darling WG. Jump landing strategies in male and female college athletes and the implication of such strategies for anterior cruciate ligamente injury. Am. J. Sports. Med. 2003;31(2):233-40.

41. Uhorchak JM, Scoville CR, Williams GN, Arciero RA, St Pirerre P, Taylor DC. Risk factors associated with noncontact injury of the anterior cruciate ligament: a prospective four-year evaluation of 859 west point cadets. Am J Sports Med. 2003;31(6):831-42.

42. Jansson A, Saartok T, Werner S, Renström P. General joint laxity in 1845 Sweedish school children of different ages: age- and gender-specific distributions. Acta Paediatr. 2004;93(9):1202-06. doi:10.1111/ j.16512227.2004.tb02749.x.

43. Shultz SJ, Shimokochi Y, Nguyen AD, Schmitz RJ, Beynnon BD, Perrin DH. Measurement of varus-valgus and internal-external rotation knee laxities in vivo Part II: relationship with anterior-posterior and general joint laxity in males and females. J. Orthop. Res. 2007;25(8):989-96. doi:10.1002/jor.20398.
44. Curtis CK, Laudner KG, McLoda TA, McCaw ST. The role of shoe design in ankle sprain rates among collegiate basketball players. J Athl Train. 2008; 43(3):230-33. doi:10.4085/1062-6050-43.3.230.

45. Wright IC, Neptune RR, Van Den Bogert AJ, Nigg BM. The influence of foot positioning on ankle sprains. J Biomech. 2000;33(5):513-19. doi:10.1016/ S0021-9290(99)00218-3.

Recebido: $12 / 03 / 2012$

Received: 03/12/2012

Aprovado: 10/12/2012

Approved: 12/10/2012 